\title{
Comunicação como instrumento de humanização do cuidado de enfermagem: experiências em unidade de terapia intensiva
}

\section{Communication as a tool of humanization of nursing care: experiences in intensive care unit}

\section{Comunicación como instrumento de humanización de la atención de enfermería: experiencias en la unidad de cuidados intensivos}

\author{
Edison Luiz Devos Barlem', Daniele Portella do Nascimento Rosenhein", \\ Valéria Lerch Lunardi" ${ }^{\prime \prime}$, Wilson Danilo Lunardi Filho ${ }^{\text {IV }}$
}

\section{RESUMO}

O presente estudo surgiu de questionamentos sobre a relevância da comunicação como instrumento para humanizar o cuidado de enfermagem na Unidade de Terapia Intensiva (UTI). Assim, o objetivo foi conhecer como pacientes perceberam 0 processo de comunicação implementado pela equipe de enfermagem, identificar quais as percepções sobre os cuidados prestados pela equipe de enfermagem e situações vivenciadas neste ambiente relacionadas ao processo de comunicação. Para tanto, foi realizada uma pesquisa qualitativa, por meio de entrevista semi-estruturada com sete pacientes que estiveram internados em UTI geral de um Hospital Universitário do extremo sul do Rio Grande do Sul, no período de janeiro de 2004 a junho de 2006, abordando aspectos referentes ao cuidado recebido, com atenção especial ao processo de comunicação. A idade dos entrevistados variou de 21 a 73 anos. Após a análise das falas, emergiram duas categorias: a vivência na UTI, seus significados e as necessidades de comunicação e UTI: potencial ambiente de cuidado. Os resultados permitem concluir que os cuidados recebidos na UTI foram satisfatórios e há o reconhecimento da preocupação da equipe de enfermagem em se comunicar com o paciente, valorizando o olhar, a presença e o toque.

Palavras chave: Unidades de terapia intensiva; Comunicação; Cuidados de enfermagem; Cuidados intensivos.

\section{ABSTRACT}

The present study appears up to the questioning involving the relevance of the communication as instrument to humanizing the nursing care at the Intensive Therapy Unit (ITU). The objective is to know how the patients noticed the communication process implemented by the nursing staff into that ambient and identifying which are their perceptions about the received cares done by the nursing staff and lived situations at that ambient related to the communication process settled down by the nursing staff. So that, it was done a qualitative research through semistructured interview with seven patients that were into at the general ITU of an university hospital the extreme south of Rio Grande do Sul, from January 2004 to J une 2006, about aspects referents to the received cares with special attention to the communication process. The age of respondents ranged from 21 to 73 years. After reviewing the speech, there were two categories: the experience in the ITU, their meanings and the needs of communication and ITU: potential environment of care. The present results permitted to conclue that the most of the customers consider that the recived care at the ITU were satisfactory and recognizing that the nursing staff worries trying to attempt of communicating with the patient, and suppling a good value for looking, presence and touching.

Key words: Intensive Care Units; Communication; Nursing Care; Intensive Care.

\section{RESUMEN}

El presente estudio surgió de cuestionamientos sobre la relevancia de la comunicación como instrumento para humanizar el cuidado de enfermería en la unidad de terapia intensiva

Enfermeiro, Aluno do Mestrado em Enfermagem da Fundação Universidade Federal do Rio Grande. E-mail: ebarlem@gmail.com

" Enfermeira, Ex-bolsista PIBIC/CNPq. E-mail: danikkaportella@hotmail.com .

III Enfermeira, Doutora em Enfermagem, Professora Associada I do Departamento de Enfermagem da Fundação Universidade Federal do Rio Grande. Pesquisadora IB do CNPq. E-mail: vlunardi@terra.com.br.

IV Enfermeiro, Doutor em Enfermagem. Professor Associado I do Departamento de Enfermagem da FURG, Pesquisador II do CNPq. E-mail: lunardifilho@terra.com.br . 
(UTI). Así, el objetivo fue conocer como pacientes perciben el proceso de comunicación implementado por el equipo de enfermería, identificar cuales son las percepciones sobre los cuidados prestados y las situaciones vivenciadas en este ambiente relacionadas al proceso de comunicación. Para tanto, fue realizada una investigación cualitativa, por medio de entrevista semi-estructurada con siete pacientes que estuvieran internados en una UTI general de un hospital universitario del extremo sur de Rio Grande do Sul, a partir de enero de 2004 a junio de 2006, abordando aspectos referentes al cuidado recibido, con especial atención al proceso de comunicación. La edad

\section{NTRODUÇÃO}

O paciente internado em Unidade de Terapia Intensiva (UTI) encontra-se comumente com sérios comprometimentos orgânicos ou em risco de vir a tê-los. Isto requer assistência contínua e permanente e um aparato tecnológico considerável, com vistas à recuperação e manutenção de suas condições de saúde e da sua qualidade de vida, quando da melhora do seu quadro clínico. Freqüentemente, por estar internado em uma unidade com essas características, o paciente pode encontrar-se em situação de profundo isolamento ${ }^{(1)}$.

Quanto mais intensivo é o tratamento, menos profundo e efetivo parece ser o contato da equipe de enfermagem que, muitas vezes, pouco toca, conversa e ouve o ser humano que está à sua frente. Quanto mais isolado está o paciente, menos a equipe parece interagir com ele. Quanto mais ele precisa ser entendido e entender, menos parece ser compreendido e menor ser sua compreensão do que se passa consigo $^{(1,2)}$.

As possíveis justificativas para esta ocorrência parecem decorrer de que a assistência em UTI ainda vem sendo, fundamentalmente, orientada pelo modelo biomédico, cuja atenção está voltada principalmente para a condição patológica e para os procedimentos técnicos e terapia medicamentosa, em detrimento da integralidade do cuidado, sem a necessária atenção aos sentimentos e percepções do doente e de seus familiares e da forma como vivenciam a situação saúde-doença ${ }^{(2-4)}$.

Entretanto, a razão do trabalho da de los encuestados oscilaban entre 21 a 73 años. Después de revisar el discurso, hay dos categorías: la experiencia en la UTI, sus significados y las necesidades de comunicación y la UTI: potencial de la atención. Los resultados permiten concluir que los cuidados recibidos en UTI fueran satisfactorios y reconocen la preocupación del equipo de enfermería en comunicarse con el paciente, valorizando la mirada, la presencia y el toque.

Palabras clave: Unidades de Terapia Intensiva; Comunicación; Atención de Enfermería; Cuidados Intensivos.

enfermagem é o cuidado terapêutico, vislumbrando uma assistência segura, integral e de qualidade, com o uso dos sentidos e sensibilidade, observando sistematicamente tanto o paciente como o ambiente $e^{(5)}$. Daí a importância da qualidade da comunicação estabelecida, sob a qual é realizado o trabalho em saúde a partir das relações humanas, seja com pacientes ou com a equipe. Logo, o processo de comunicação está inteiramente inserido nas ações da enfermagem, cabendo especialmente ao enfermeiro interpretar, desvendar e entender o significado das mensagens que os pacientes enviam, para que possa instituir um plano de cuidados apropriado e coerente às suas necessidades.

A intenção básica da comunicação é melhorar o entendimento da realidade, as relações com os outros e modificar-se a si mesmo e aos fatos. É essencial o profissional compreender que a maneira como o paciente percebe os acontecimentos à sua volta influencia a sua conduta mais do que a realidade da situação em $\mathrm{si}^{(1,5-8)}$.

Considerando o processo de comunicação, particularmente, em UTI, é importante considerar que nesse local, a experiência dos pacientes, na maioria das vezes, é de um tratamento invasivo, agressivo e até mesmo doloroso. A vivência na UTI é descrita pelos pacientes como permeada pela solidão $e$ desamparo, em que se sentem controlados por meio de máquinas pela equipe de saúde e hesitantes diante da incerteza de seus destinos; desconfortáveis fisicamente e inseguros emocionalmente com o desencadeamento de 
reações que variam do silêncio ao choro e à agitação(6).

Embora seja preconizado que o acolhimento em uma UTI seja silencioso e discreto, comumente, o paciente recebe uma grande quantidade de estímulos sensoriais: sente-se atingido pelos sons emitidos pelos outros pacientes - respirações ruidosas, palavras, gemidos e gritos; as vozes dos membros da equipe, o ambiente estranho e geralmente hostil em sua volta e demais fatores ambientais, tais como os alarmes, a iluminação contínua e a climatização local ${ }^{(9)}$. Assim, a internação em uma UTI rompe bruscamente com o seu modo de viver, interferindo em suas relações, seus papéis e seus padrões de comunicação.

Devido à gravidade em que se encontra, o paciente internado em UTI nem sempre é respeitado como sujeito apto a escolher, decidir, opinar e com direito à expressão e à informação. Por vezes, por ter seu contato visual e verbal prejudicado com os profissionais, é submetido quase que totalmente àqueles que dele cuidam, não sendo atendido como uma pessoa singular, porém, transformado em receptáculo de cuidados técnicos e intensivos, que sofre experimentos, fragmentado em problemas e tratado com imparcialidade ${ }^{(4,10)}$.

É necessário olhar o paciente por outro ponto de vista, como sujeito e cidadão que carrega consigo, para dentro das instituições, seus desejos, temores, sofrimentos e expectativas $^{(10)}$. Ainda, muitos pacientes sentem-se vulneráveis, angustiados e com medo, uma vez que a internação em UTI não apenas vem impregnada de mitos e fantasias, mas também da possibilidade constante da ocorrência da morte. Desse modo, o cuidado holístico abarca um processo comunicativo eficaz entre enfermeiro/equipe de enfermagempaciente, que se concretiza de diferentes formas ${ }^{(5)}$.

A comunicação nesse sentido pode ser o alicerce das relações entre os profissionais e os pacientes $^{(7)}$. Por meio dos vínculos estabelecidos, podemos esperar um paciente que coopera no dia a dia para o sucesso da sua recuperação ou indiferente, diante da sua realidade, e com um nível de frustração patológico, que formará uma grande barreira em todo o seu processo de cura, desencadeando ou, até mesmo, abreviando o seu processo de morte ${ }^{(9)}$. Portanto, a comunicação é uma das competências que precisa ser utilizada pelo enfermeiro e sua equipe, a fim de expandir a habilidade de entender as mensagens implícitas ou explícitas, que permeiam a relação com o paciente ${ }^{(8)}$.

Assim, considerando a relevância da comunicação como parte significativa do cuidado de enfermagem, em especial no contexto da terapia intensiva, e preocupados com a sua humanização e qualidade, tivemos como objetivo: identificar como pacientes que já foram internados em uma UTI perceberam o processo de comunicação implementado pela equipe de enfermagem, por meio de suas percepções sobre os cuidados que lhes foram prestados, durante a internação. Reafirmamos a idéia de que a excelência no cuidar também é baseada em comunicações mais efetivas, nos mais diversos ambientes de trabalho da enfermagem, sendo necessárias para atingir esse fim, a reorganização e adaptação do processo de trabalho em suas dimensões teórico-práticas ${ }^{(11)}$.

\section{O CAMI NHO METODOLÓGI CO}

Pesquisa qualitativa de abordagem descritiva exploratória, realizada em uma UTI geral de um Hospital Universitário (HU) do extremo sul do Rio Grande do Sul no período de junho a dezembro de 2006.

Após a autorização para a realização da pesquisa pela direção e chefia de enfermagem do $\mathrm{HU}$ e encaminhamento e aprovação do projeto pelo respectivo Comitê de Ética em Pesquisa na Área da Saúde - CEPAS/FURG (Protocolo 051/2006), foram seguidos os seguintes passos: consulta ao livro de registros das altas da UTI, seleção aleatória e análise dos prontuários, convite e agendamento de entrevistas.

Para a realização do estudo foram definidos critérios para a seleção dos sujeitos, tais como: terem sido internados e recebido alta da UTI Geral - Adulto do Hospital Universitário, entre janeiro de 2004 e junho de 2006; residirem no perímetro urbano do município; terem a idade mínima de 18 anos; estarem orientados no tempo e espaço; terem 
capacidade de comunicar-se e concordarem em participar do estudo.

Diversas tentativas de busca de possíveis participantes foram realizadas por ligações telefônicas e visitas aos endereços que constavam nos prontuários. No entanto, várias dessas pessoas já haviam mudado de endereço ou falecido, o que resultou num total de sete sujeitos que concordaram em participar do estudo.

As entrevistas foram realizadas a partir da solicitação de consentimento livre e esclarecido, sendo gravadas e conduzidas por um roteiro com questões abertas, enfocando percepções dos pacientes em relação à comunicação com a equipe de enfermagem durante a realização de procedimentos, dificuldades, facilidades, conteúdo dos diálogos; percepção sobre os cuidados: privacidade, isolamento, necessidades sentidas e manifestadas, atenção, tempo da equipe de enfermagem direcionado diretamente ao paciente, presença da família, sentimentos, vivências, dificuldades e aspectos que poderiam ser melhorados no ambiente da UTI.

Para a análise dos dados, adotamos uma abordagem de análise cíclica ${ }^{(12)}$, que iniciou com a desmontagem dos textos resultantes das transcrições, seguidas de sua categorização e posterior argumentação acerca do fenômeno emergente.

A análise das falas dos entrevistados possibilitou conhecer como a comunicação fez parte dos cuidados e ainda "descobrir o que [estava] por trás dos conteúdos manifestos, indo além das aparências do que [estava] sendo comunicado"(13). Deste modo, pudemos "compreender os dados obtidos, confirmando ou não os pressupostos do estudo e/ou responder às questões formuladas, ampliando o conhecimento acerca do assunto pesquisado"(13), conforme as categorias que serão apresentadas a seguir.

\section{APRESENTAÇÃO DOS RESULTADOS}

Dos sete entrevistados, um era do sexo masculino e seis do sexo feminino, com idades variando de 21 a 73 anos. As causas primárias de internação foram variadas: tentativa de suicídio, hipertensão, choque anafilático, convulsão, cetoacidose diabética e complicações durante pneumonia. Para alguns dos entrevistados, essas foram as doenças de base, logo desenvolvendo outras complicações durante a internação.

A seguir, serão enfocados, inicialmente, significados e necessidades de comunicação percebidas pelos pacientes em suas vivências na UTI, e a UTI como um potencial ambiente de cuidado, em que o modo como as práticas são implementadas e as relações instituídas e comunicadas podem caracterizar tais práticas como de cuidado ou não.

\section{A vivência na UTI, seus significados e as necessidades de comunicação}

A partir dos relatos, foi possível caracterizar momentos distintos da comunicação na internação na UTI. Na chegada, - paciente é abordado por diferentes profissionais da equipe de saúde, que o conectam a vários instrumentos, lhe dão medicações, iniciando uma comunicação mediada por ordens e estímulos verbais: "abra os olhos", "calma, já vai passar", "você sabe onde está"? No momento em que o paciente se percebe na UTI, muitas vezes, reage de maneira singular e demonstra, por meio de ações de inconformidade, o impacto desta nova realidade:

Quando eu acordei, eu entendi que eu tava numa UTI, (...) comecei a me arrancar tudo (P3).

Em tais situações, a tendência da equipe é procurar orientá-lo e tranqüilizá-lo:

Você está na UTI da... Está tudo bem! Ou calma! Você tem que se acalmar, não faz assim..." (P1).

Este é um momento percebido como importante e que lhe ajuda a situar-se, quanto ao tempo e espaço, minimizando os conflitos gerados pela desorientação.

Por sua vez, o termo UTI já evoca uma gama de significados, principalmente, quando o paciente nunca tivera contato anterior com este ambiente:

Pra mim, eu não ia mais sair dali, eu ia morrer. Puxa, se eu tô na UTI, então, eu tô morrendo, sabe (P2).

Portanto, estar numa UTI denota encontrar-se entre a vida e a morte, podendo representar uma ida sem volta. Além disso, os equipamentos, o número de profissionais em 
torno de si, os sons e os ruídos, o modo como eventualmente se dá sua admissão e o não entendimento sobre o seu estado de saúde e dos demais pacientes internados fazem com que o paciente experimente sentimentos de medo e insegurança, ressaltando ainda mais a existência deste estigma de associação de UTI com morte ${ }^{(3)}$.

Daí a necessidade de comunicação eficaz e uma relação de empatia, durante os encontros que, embora breves, necessitam ser conduzidos com honestidade e sensibilidade. Para tanto, é preciso considerar a singularidade, os valores e crenças dos pacientes e utilizar-se de uma linguagem clara, de acordo com o seu nível de compreensão, permitindo uma avaliação mais fidedigna de seus problemas e necessidades ${ }^{(14)}$.

O paciente, ao perceber-se internado em uma UTI, pode experimentar também sentimentos como solidão e isolamento:

Quando a gente chega num hospital, se descobre dentro de uma UTI, tu te sente carente, tu te sente abandonada... (P2).

A promoção de seu acolhimento torna-se possível por intermédio da comunicação, sabendo ouvi-lo e mostrando-se disposto a ajudá-lo a enfrentar as situações que está vivenciando:

Mas eu tive amparo. Tiveram zelo... Elas são muito preparadas pra estar lá. Eu melhorei muito pelo zelo delas. Porque podia trocar de turno, mas a atenção dada pra mim era a mesma (P2).

Desse modo, as posturas e atitudes profissionais representam formas de comunicação que, quando terapêuticas, se constituem em instrumento útil para ajudá-lo a superar os sentimentos decorrentes destas situações e a recuperar o ânimo e o bemestar ${ }^{(15)}$ :

...e sempre ela vinha e me falava: olha tu tens que acordar. Vamos lá! Mas meu olho pesava... (P4).

Os profissionais necessitam ter sempre presente que, em seu exercício diário, a comunicação verbal é reforçada pela comunicação não-verbal, reconhecida como "a dimensão da comunicação que qualifica as relações, permitindo a demonstração dos sentimentos e a verificação da coerência dos nossos gestos e posturas no estar com as pessoas"(16). Assim, podemos dizer que há conexão entre ambas, comunicação verbal e não-verbal, demonstrando a efetividade da comunicação ${ }^{(16)}$, ainda que o profissional esteja apenas fazendo-se presença:

Olha, sempre que eu olhava pra elas, elas estavam sempre de olho em mim... perto. Daí eu via que não estava jogado (P1).

Portanto, a comunicação como um instrumento de cuidado, tanto a verbal como a não verbal, estimula a reação do paciente, especialmente em uma UTI, podendo ajudá-lo a superar problemas, a relacionar-se com os demais e a ajustar-se ao que muitas vezes não pode ser mudado.

A comunicação é um processo complexo e demanda uma aplicação inteligente de princípios, em que o profissional cria, fala e age de modo a iniciar modificações que visam o bem estar do paciente, permitindo sua segurança e satisfação:

Elas sempre chegavam e perguntavam: tu tá bem? Quer alguma coisa? Era só eu ficar um pouquinho mais quieta que alguém chegava ou verificava os sinais, pra ver se tava tudo certinho (P5).

A compreensão da UTI como um local de cuidado, de recuperação da saúde, de sinônimo de vida e de oposição à morte ${ }^{(17)}$ pode estar fortemente relacionada ao modo como os trabalhadores de enfermagem comunicam-se e comunicam seu fazer aos pacientes, o que confirma que, também em UTIs "na enfermagem, não há como dissociar a educação do cuidado"(18).

\section{UTI : potencial ambiente de cuidado}

Relacionar-se com pessoas não é só atentar para os aspectos físicos ou biológicos, mas abarca a humanização, uma maior responsabilidade e respeito pelo outro, pelo seu modo de ser e de agir, aspectos que se consolidam com a efetiva comunicação( ${ }^{(8)}$. A humanização do cuidado é uma temática que atualmente vem sendo muito discutida pelos profissionais de enfermagem. A comunicação mostra-se como extraordinário instrumento de humanização do cuidado de enfermagem e da assistência como um todo, especialmente, em uma UTI ${ }^{(15)}$.

A presença e o modo como a atuação 
profissional ocorre na relação com o paciente pode comunicar a ele ações de cuidado ou apenas a mera realização de tarefas e procedimentos técnicos prescritos ou, até mesmo, desinteresse:

Tinham pessoas que eram mais malandros, que eu via que estavam ali, mas que não estavam se dedicando... Eu via que trabalhavam, ali, por obrigação. (P2).

Portanto, existem trabalhadores da saúde e da enfermagem que atuam sem um suficiente comprometimento "moral" com o paciente, com a instituição e com a profissão, confirmando que "terapêuticas, procedimentos, técnicas, intervenções podem ser prescritas, não o cuidado. [...] Não se prescreve o modo de ser, não se ditam maneiras de se comportar; elas podem ser sugeridas, aconselhadas, mas não prescritas" ${ }^{\prime 19)}$.

Muitos pacientes referiram ter sofrido calados e de modo solitário com os desconfortos decorrentes das normas e rotinas impostas, aparentemente como condições para a sua recuperação:

...eu pedia minha roupa. Eu me olhava de fralda e ficava furioso. Reclamava, pedia uma roupa..."(P1).

Sofriam, também, durante a realização de procedimentos, especialmente, quando invasivos:

Dava muita aflição. Em primeiro lugar, era o tubo pra tirar a secreção do pulmão, né? Tubo pra comer... tubo para respirar oxigênio... Então, não tem condições, né? (P7)

Assim, os grandes impactos da internação referidos pelos pacientes foram relativos à perda de autonomia, à despersonalização e ao quanto foi difícil ter que se submeter às normas, rotinas e procedimentos técnicos:

$A i$, quando iam fazer aquela aspiração... Meu Deus do céu! Parece que tá arrancando a gente, na hora que puxa... Mas aquilo dá um medo... O barulho que faz... E, quando você sabe o que vai acontecer... Pior é que a gente não pode fugir... Era horrível! (P1).

Apesar da dificuldade de lidar com esta realidade imposta, percebemos que o paciente acata e submete-se às decisões da equipe de saúde e às rotinas e normas institucionais, aparentemente incorporando e assumindo a postura esperada de um "paciente":
Tu te sente meio que incapaz, porque o simples fato de tomar um banho ou escovar os dentes, ou lavar o rosto... Nada você pode fazer sozinha. Tu tem que ter uma pessoa, ali... E é a seringa e é o soro dependurado... Tu não pode te pentear sozinha. Tem que ter uma pessoa pra te pentear, né? É! São vários incômodos que você acaba ficando dependente. Se quiser ir no banheiro... Ah! Tem que chamar. Ah! Consegue a comadre... De tudo, esse é o mais complicado... É difícil!(P5).

Desse modo, mesmo que incentivado ao autocuidado, sofre por depender de outrem, sendo fundamental uma atuação profissional empática e comprometida com o seu bem-estar, oportunizando meios para que possa, ainda que parcialmente, exercer sua autonomia, minimizando os efeitos negativos de sua condição de doente e da internação.

Assim, independentemente de estar internado ou não em uma UTI, há a necessidade do reconhecimento do paciente como sujeito do cuidado e não seu mero objeto, assegurando, sempre que possível, seu direito a escolhas, à decisão, a partir do que acredita, valoriza e deseja para si; senão, transparecem raízes de um cuidado despersonalizado, centrado na execução de tarefas e procedimentos técnicos esvaziados de conteúdos de enfermagem.

Apesar da complexidade tecnológica do ambiente de uma UTI, o enfermeiro e os demais membros da equipe de enfermagem podem ser facilitadores da promoção do seu conforto e bem-estar bio-psico-sócio-espiritual, por meio da realização de determinados cuidados de enfermagem, por mais elementares que possam parecer $^{(5)}$ :

Na hora do banho, elas sempre falavam: vamos virar você, não te assusta meu velho! Não precisa ter medo, vamos ficar bonito, pra ver as visitas... Ah! O banho era bom (P1).

Com a continuidade de seu tratamento e a evolução favorável de seu quadro clínico, foram experimentadas sensações que retratam dificuldades na satisfação de muitas de suas necessidades e a importância da participação da equipe de enfermagem para amenizar tais problemas:

Eu sentia uma fome que nem sei... (P5).

Eu escrevi, no papelzinho, porque eu não podia 
falar, por causa do tubo. Sentia muita fome e frio, mas deixaram eu colocar uma meia... A mãe levou e elas colocaram [risos]...(P1).

Assim, podemos perceber que a participação e presença da família parecem ser extremamente relevantes para o bem-estar do paciente, o que não é suficientemente contemplado nem considerado como parte da integralidade do cuidado. $O$ afastamento do convívio com seus familiares constitui-se em fonte de sofrimento, agravado pelo pouco tempo permitido para a realização e permanência das visitas:

Eu olhava as pessoas da minha família só pra dizer um oi, porque tinha que ser rápido. [...] Passava o dia esperando, pra dizer só um oi (P1).

Além disso, há o surgimento de sentimentos relacionados à preocupação em deixar seus familiares "sozinhos" e pelo trauma decorrente da sua internação:

Também tive muito medo pelos meus familiares, porque eles não sabiam o que estava acontecendo, ali dentro. Eu cheguei lá, na terça à noite, e só fui acordar na quinta. Então, eles ficaram muito aflitos" (P2).

Desse modo, os horários de visita apresentam-se como momentos fundamentais indispensáveis, tendo em vista que o afastamento e separação do paciente do meio familiar podem ser fatores geradores de estresse e insegurança tanto para si como para sua família. Assim, a presença do familiar na UTI pode contribuir tanto para que o paciente enfrente os seus sentimentos de medo, insegurança, solidão, perda de autonomia e despersonalização quanto o enfrentamento pela própria família dos sentimentos que também vivencia nesta situação ${ }^{(3)}$.

Daí a relevância da ampliação dos horários de visita e até da possibilidade de permanência de um familiar junto ao paciente. Tais decisões implicam mudanças na cultura dos profissionais de saúde e na organização do trabalho em UTI: de conviverem com a presença e permanência de um outro que não um paciente ou trabalhador; um outro possivelmente atento não apenas às necessidades de seu familiar, mas à dinâmica do processo de trabalho ali desenvolvido - procedimentos, cuidados, intercorrências, manifestações verbais ou não e omissões, provavelmente contribuindo, também, para o atendimento da necessidade do paciente perceber-se realmente cuidado.

Para o paciente, não basta a execução de procedimentos tecnicamente corretos, mas que quem the preste cuidados interaja com ele e faça-o sentir-se realmente cuidado.

É uma pessoa ter carinho, né? (...) Cuidado melhor que tu pode dar pro teu paciente é uma palavra de conforto, de amor... É a principal coisa. Não adianta dar banho, dar uma comidinha boa na boca, tá? Isso aí não adianta nada!" (P7). "Porque tu tem que passar segurança pro teu paciente" (P2).

Assim, para o paciente, cuidar é dar atenção, estar atento, ouvi-lo, explicar tudo o que ele queria saber. Tanto para o enfermeiro como para os demais profissionais, resta aguçar a percepção e ir ao encontro dos anseios e necessidades do paciente, sabendo que em todas as suas ações, com palavras, sem palavras, com o corpo, com sua presença ou não, estão comunicando-se com ele e comunicando uma forma de ser e de fazer, com repercussões para o seu modo de sentir-se ou de não sentir-se cuidado. É preciso “dar à comunicação um significado mais amplo por meio da interação, possibilitando o envolvimento, o conhecimento do outro"(20) e também o permanente conhecimento de si, a partir da reflexão sobre o seu modo de ser e de fazer.

\section{CONSI DERAÇÕES FINAIS}

Com a realização deste estudo, foi possível evidenciar que a internação em uma UTI ainda desencadeia sentimentos de insegurança e sofrimento tanto para o paciente como para a sua família. Os membros da equipe de enfermagem, em sua maioria, apresentam estratégias de comunicação, sejam verbais, para-verbais ou não verbais para lidar com os pacientes, mesmo quando sedados, durante a realização dos diferentes procedimentos e cuidados. Para o paciente, a comunicação apresenta-se como um componente do cuidado que existe como forma de transmitir segurança, respeito e carinho, para muito além do conteúdo das explicações técnicas sobre o seu estado de saúde.

Assim, comunicar-se com o paciente em 
uma UTI é mais do que Ihe explicar, ainda que detalhadamente sobre um procedimento ou uma medicação: é estar ali por ele e com ele, dar-Ihe atenção e interagir. Percebemos que aqueles que participaram deste estudo, muito além do intensivismo necessário para a sua recuperação manifestaram o desejo por um cuidado humanizado, somente possível quando acompanhado pelo olhar atento, pelo toque, pela empatia e pelo fazer-se presença dos profissionais.

Apesar de a maioria dos clientes considerarem que os cuidados prestados pela equipe de enfermagem da UTI foram satisfatórios e reconhecerem a preocupação da equipe de enfermagem, na tentativa de se comunicarem consigo, sugerimos como relevante a intensificação da presença de um familiar neste ambiente, de modo a contribuir para o enfrentamento do sofrimento e desconforto vivenciados tanto pelo paciente como por sua família, bem como para contemplar a possibilidade do exercício de sua autonomia e de sua singular condição de pessoa.

\section{REFERÊNCI AS}

1. Ferreira MIPR. A comunicação entre a equipe de saúde e o paciente em coma: dois mundos diferentes em interação [dissertation]. Florianópolis: Universidade Federal de Santa Catarina/UFSC; 2000. 106 p.

2. Gonzales RF, Branco R. Relação com o paciente: teoria, ensino e pratica. Rio de J aneiro: Guanabara Koogan; 2003.

3. Lemos RCA, Rossi LA. O significado cultural atribuído ao centro de terapia intensiva por clientes e seus familiares: um elo entre a beira do abismo e a liberdade. Rev Latino-am Enfermagem. 2002; 10(3):345-57.

4. Nascimento ERP, Trentini M. O cuidado de enfermagem na unidade de terapia intensiva (UTI): teoria humanística de Paterson e Zderad. Rev Latino-am Enfermagem. 2004; 12(2):250-7. 5. Oriá MOB, Moraes LMP, Victor JFA. Comunicação como instrumento do enfermeiro para o cuidado emocional do cliente hospitalizado. Rev. Eletr. Enf. [Internet]. 2004 [cited 2008 dec 31];6(2):292-7. Available from: http://www.fen.ufg. br/revista/revista6_2/comu nica.html.
6. Zinn GR, Silva MJP, Telles SCR. Comunicar-se com o paciente sedado: vivência de quem cuida. Rev Latino-am Enfermagem. 2003; 11(3):326-32.

7. Tigulini RS, Melo MRAC. A comunicação entre enfermeiro, família e paciente crítico. In: Anais do 8 o Simpósio Brasileiro de Comunicação em Enfermagem [Internet]; 2002 Maio 02-03; São Paulo, Brasil. 2002 [cited 2008 dec 31]. Available from: http://www. proceedings. scielo.br/pdf/sibracen/ n8v2/v2a113.pdf.

8. Silva LMG, Brasil VV, Guimarães HCQCP, Savonitti BHRA, Silva MJP. Comunicação nãoverbal: reflexões acerca da linguagem corporal. Rev Latino-am Enfermagem. 2000; 8(4):52-8.

9. Andrade MTS. Guias práticos de enfermagem: cuidados intensivos. Rio de Janeiro: McGrall-Hill; 2002.

10. Boemer MR, Corrêa AK. Repensando a relação do enfermeiro com o doente: o resgate da singularidade humana. In: Gonzáles RF, Branco R. Relação com o paciente: teoria, ensino e prática. Rio de Janeiro: Guanabara Koogan; 2003, p. 263-9.

11. Barbosa EMA, Brasil VV. Boletim informativo em UTI: percepção de familiares e profissionais da saúde. Rev. Eletr. Enf. [Internet]. 2007 [cited 2008 mar 17];9(2):315-28. Available from:

http://www. fen.ufg. br/revista/v9/n2/v9n2a03.htm 12. Moraes R. Uma tempestade de luz: a compreensão possibilitada pela análise textual discursiva. Ciência \& Educação. 2003;9(2): 191211.

13. Minayo MCS, Deslandes SF, Neto OC, Gomes R. Pesquisa social: teoria, método e criatividade. Rio de Janeiro: Vozes; 1998.

14. Oliveira PS, Nóbrega MML, Silva AT, Filha MOF. Comunicação terapêutica em enfermagem revelada nos depoimentos de pacientes internados em centro de terapia intensiva. Rev. Eletr. Enf. [Internet]. 2005 [cited 2006 jul 27]; 7(1):54-63. Available from: http://www.fen.ufg.br/revista7 1/original 02.htm. 15. Camargos AT, Dias LO. Comunicação: um instrumento importante para humanizar o cuidado de enfermagem em unidade de terapia intensiva. In: Anais do 8o Simpósio Brasileiro de Comunicação em Enfermagem [Internet]; 2002 Maio 02-03; São Paulo, Brasil. 2002 [cited 2008 
Barlem ELD, Rosenhein DPN, Lunardi VL, Lunardi Filho WD. Comunicação como instrumento de humanização do cuidado de enfermagem: experiências em unidade de terapia intensiva. Rev. Eletr. Enf. [Internet]. 2008; 10(4): 1041-9. Available from: http://www.fen.ufg. br/revista/v10/n4/v10n4a16.htm.

dec 31]. Available from:

http://www.proceedings. scielo.br/pdf/sibracen/

n8v1/v1a032.pdf.

16. Inaba LC, Silva MJP, Telles, SCR. Paciente crítico e comunicação: visão de familiares sobre sua adequação pela equipe de enfermagem.

Rev. esc. enferm. USP. 2005;39(4):423-9.

17. Severo GC, Girardon-Perlin NMO. Estar internado em unidade de terapia intensiva: percepção de pacientes. Scientia Medica. 2005; 15(1): 21-9.

18. Nascimento KC, Erdmann AL. Cuidado transpessoal de enfermagem a seres humanos em unidade crítica. Rev. Enferm. UERJ. 2006; 14(3): 133-41.

19. Waldow VR. Cuidar: expressão humanizadora da enfermagem. Rio de Janeiro: Vozes; 2006.

20. Oliveira MMC, Almeida CB, Araújo TL, Galvão MTG. Aplicação do processo de relação interpessoal de Travelbee com mãe de recémnascido internado em uma unidade neonatal. Revista Rev. esc. enferm. USP. 2005; 39(4): 430-6.

Artigo recebido em 14.08.07.

Aprovado para publicação em 31.12.08. 